\title{
TPX2 is a prognostic marker and promotes cell proliferation in neuroblastoma
}

\author{
YUHKI KOIKE $^{1 *}$, CHENGZENG YIN $^{1 *}$, YUKI SATO $^{1}$, YUKA NAGANO $^{1}$, AKIRA YAMAMOTO $^{1}$, \\ TAKAHITO KITAJIMA $^{1,2}$, TADANOBU SHIMURA ${ }^{1}$, MIKIO KAWAMURA ${ }^{1}$, KOHEI MATSUSHITA ${ }^{1}$, \\ YOSHINAGA OKUGAWA ${ }^{1,2}$, KEISHIRO AMANO ${ }^{3}$, KOHEI OTAKE ${ }^{4}$, YOSHIKI OKITA $^{1}$, MASAKI OHI $^{1}$, \\ MIKIHIRO INOUE ${ }^{1}$, KEIICHI UCHIDA ${ }^{1}$, MASAHIRO HIRAYAMA ${ }^{3}$ and YUJI TOIYAMA ${ }^{1}$ \\ ${ }^{1}$ Department of Gastrointestinal and Pediatric Surgery, Division of Reparative Medicine, Institute of Life Sciences, \\ Mie University Graduate School of Medicine; ${ }^{2}$ Department of Genomic Medicine, Mie University Hospital; \\ ${ }^{3}$ Department of Pediatrics, Mie University Graduate School of Medicine, Tsu, Mie 514-8507; \\ ${ }^{4}$ Department of Surgery, Mie Prefectural General Medical Center, Yokkaichi, Mie 510-0885, Japan
}

Received August 26, 2021; Accepted February 7, 2022

DOI: $10.3892 / \mathrm{ol} .2022 .13256$

\begin{abstract}
Targeting protein for Xenopus kinesin-like protein 2 (TPX2) is upregulated in various tumors, and several studies have demonstrated the role of TPX2 as a prognostic marker in cancer. However, the function of TPX2 in neuroblastoma (NB) has not been completely elucidated. In the present study, the clinical significance and functional role of TPX2 in NB was investigated. The Therapeutically Applicable Research to Generate Effective Treatments (TARGET)-NB dataset was used. A total of 43 patients with NB were enrolled in the present study as the validation set. After evaluating the prognostic role of TPX2, the combined predictive effect of TPX2 and MYCN proto-oncogene bHLH transcription factor (MYCN) gene amplification was assessed. Double immunofluorescence staining for TPX2 and N-Myc was used to analyze colocalization, and multiple cell function tests were performed by means of in vitro experiments to elucidate the functional role of TPX2 using RNA interference technology in NB cell lines. In
\end{abstract}

Correspondence to: Dr Yuji Toiyama or Dr Yuhki Koike, Department of Gastrointestinal and Pediatric Surgery, Division of Reparative Medicine, Institute of Life Sciences, Mie University Graduate School of Medicine, 2-174 Edobashi, Tsu, Mie 514-8507, Japan

E-mail: ytoi0725@clin.medic.mie-u.ac.jp

E-mail:koikyon@gmail.com

*Contributed equally

Abbreviations: TPX2, targeting protein for Xenopus kinesin-like protein 2; NB, neuroblastoma; TARGET, Therapeutically Applicable Research to Generate Effective Treatments; siRNA, small interfering RNA; ROC, receiver operating characteristic; OS, overall survival; AURKA/Aurora-A, aurora kinase A

Key words: neuroblastoma, TPX2, biomarker, prognosis, MYCN both the TARGET-NB set and the validation set, it was found that upregulated of TPX2 was significantly associated with poor overall survival (OS) in patients with NB. The expression of TPX2 was higher in NB patients with MYCN gene amplification, and NB patients with high TPX2 expression and MYCN gene amplification had the poorest OS compared with patients with low TPX2 expression or a single copy of MYCN. In vitro experiments indicated that TPX 2 positively regulated cell proliferation and the cell cycle, and promoted cell survival by increasing the resistance to apoptosis. The colocalization of TPX2 with N-Myc in NB cells and tissue was observed. The findings of the present study indicate that TPX2 plays an oncogenic role in NB development and may be a potential prognostic indicator in patients with NB.

\section{Introduction}

Neuroblastoma (NB) is a tumor that originates from precursor cells in the nervous system and is the most common extra-cranial solid tumor in children $(1,2)$. The 5-year survival rate in 2010 for children <1 year old was $95 \%$ in the United States, and the understanding of this disease has advanced tremendously in the past decade (2). Despite the ongoing comprehensive research into this disease, NB still accounts for $6 \%$ of all pediatric malignancies in the United States $(3,4)$ and $11 \%$ of all pediatric cancer-related deaths in patients $<15$ years old (2). Furthermore, the long-term survival of high-risk patients with NB is $<50 \%$ due to the heterogeneous, aggressive and relapse-prone nature of these tumors $(5,6)$. Therefore, the identification of novel prognostic markers for NB is required.

Targeting protein for Xenopus kinesin-like protein 2 (TPX2; also known as C20orf1, C20orf2, DIL-2 and p100) is required for microtubule formation and regulates cell movement during key biological processes $(7,8)$, such as proliferation, apoptotic processes and cell division (9-11). An oncogenic role for TPX2 has been demonstrated in multiple malignancies, including gastric cancer, colorectal carcinoma, 
hepatocellular carcinoma and bladder cancer (12-15). In a lung cancer study, increased TPX2 was observed during the establishment of drug tolerance and was maintained into acquired resistance (16). A recent study suggested that TPX2 may be a prognostic marker to stratify high-risk NB (17). However, the function of TPX2 in NB has not been completely elucidated.

The current prognostic assessment of NB is based on the NB Risk Classification System, including The International NB Staging System stage, tumor histology, age at diagnosis, chromosome copy number alteration and MYCN proto-oncogene bHLH transcription factor (MYCN) gene status (18-20). Combined assessment of TPX2 expression and MYCN gene amplification may be a prognostic marker to stratify high-risk NB patients. In the present study, the prognostic significance of TPX2 combined with MYCN gene amplification in patients with NB was evaluated and the functional role of TPX2 in NB cell lines was elucidated.

\section{Materials and methods}

Patients and sample collection. NB tissue samples and data of 43 patients with NB who underwent surgical resection between March 1980 and February 2010 at Mie University Hospital (Tsu, Japan) were obtained retrospectively from the Department of Pathology and from patient records. All patients were managed according to the Japan NB Study Group protocol (21). The median patient age was 8 months (ranging from 1 month to 8 years) and $23.3 \%$ of the patients were female. The clinicopathological characteristics of the patients are listed in Table I. The amplification status of MYCN from 39 of 43 patients was obtained from the Department of Pathology, Mie University Hospital, as assessed by fluorescence in situ hybridization. The protocol for the present research project was approved (approval no. 1464) by the Institutional Review Board of Mie University Hospital. Informed consent was obtained from the parents/guardians of the patients using the opt-out scheme.

Survival information and gene expression data for 247 NB samples were also obtained from the Therapeutically Applicable Research to Generate Effective Treatments (TARGET) project (https://ocg. cancer.gov/programs/TARGET/data-matrix) conducted in November 2018. The TARGET-NB dataset was used for survival analysis and association between TPX2 expression and other gene expression. The clinicopathological characteristics of the TARGET-NB data set are listed in Table SI.

Cell lines and culture. The MYCN-amplified IMR-5 cell line and the MYCN-non-amplified KP-N-SI(FA) cell line were acquired from the Cell Resource Center of Biomedical Research, Institute of Development, Aging and Cancer (Tohoku University). Both cell lines were maintained in RPMI-1640 medium (cat. no. 30264-56; Nacalai Tesque, Inc.) supplemented with $10 \%$ fetal bovine serum (cat. no. s1580-500; Biowest), glutamine (2 mmol/l), penicillin $(100,000 \mathrm{U} / \mathrm{l})$, streptomycin $(100 \mathrm{mg} / \mathrm{l})$ and gentamicin $(40 \mathrm{mg} / \mathrm{l})$ at $37^{\circ} \mathrm{C}$ in a $5 \% \mathrm{CO}_{2}$ atmosphere. The cell lines were tested and authenticated.

Total RNA extraction and cDNA synthesis. Total RNA was extracted from NB cells and tissues using the RNeasy mini kit (cat. no. 217004) and RNeasy FFPE kit (cat. no. 217504; both from Qiagen $\mathrm{GmbH}$ ) in accordance with the manufacturer's instructions. RNA quality and concentration were determined using a Denovix ${ }^{\circledR}$ DS-11+ spectrophotometer (DeNovix, Inc.). cDNA was synthesized from $5 \mu \mathrm{g}$ of total RNA with random hexamer primers, dNTPs, $5 \mathrm{X}$ buffer and SuperScript $^{\circledR}$ III Reverse Transcriptase (cat. no. 8080-044; Invitrogen; Thermo Fisher Scientific, Inc.) as previously described (22).

Reverse transcription-quantitative PCR (RT-qPCR). RT-qPCR analyses of NB cells and tissues were conducted using the Power SYBR Green PCR Master Mix (cat. no. 4367659; Applied Biosystems; Thermo Fisher Scientific, Inc.) and the Applied Biosystems 7500 Real-Time PCR System (Applied Biosystems; Thermo Fisher Scientific, Inc.) in accordance with the manufacturer's instructions and as previously described (22). The qPCR cycling conditions were as follows: $95^{\circ} \mathrm{C}$ for $10 \mathrm{~min}$, followed by 40 cycles of $15 \mathrm{sec}$ at $95^{\circ} \mathrm{C}$ and $60 \mathrm{sec}$ at $60^{\circ} \mathrm{C}$. Primers for TPX2 and GAPDH mRNAs were as follows: TPX2 forward, 5'-TGAGGCAGCCATATCAAG AA-3' and reverse, 5'-TGGCACATCTCTTGGCTTTC-3'; and GAPDH forward, 5'-GGAAGGTGAAGGTCGGAGTC-3' and reverse, 5'-AATGAAGGGGTCATTCATGG-3'. Relative expression levels of TPX 2 mRNA were calculated by normalization to the levels of endogenous GAPDH mRNA using the $2^{-\Delta \Delta C q}$ method (23). RT-qPCR assays were performed in triplicate for each sample and the mean value was calculated.

TPX2 RNA interference. TPX2-specific small interfering RNA (siRNA) (cat. no. 4392429; Silencer ${ }^{\circledR}$ Predesigned siRNA) and negative control siRNA (cat. no. 4390844; Silencer Negative Control siRNA) were purchased from Ambion ${ }^{\circledR}$; Thermo Fisher Scientific, Inc. The sequences of TPX2 siRNA were as follows: Sense, 5'-GGAAAGUGAACUUUACAUCUtt-3' and antisense, 5'-AGAUGUAAAGUUCACUUCCtt-3'. Cells were seeded in six-well culture plates at $2 \times 10^{5}$ cells per well in $2 \mathrm{ml} \mathrm{RPMI-1640.} \mathrm{Cells} \mathrm{were} \mathrm{cultured} \mathrm{for} 24 \mathrm{~h}$ and then incubated with siRNA oligonucleotides using Lipofectamine ${ }^{\circledR}$ RNAiMAX Reagent (cat. no. 13778-150) and OptiMEM ${ }^{\circledR}$ I (cat. no. 31985-062) (both Invitrogen; Thermo Fisher Scientific, Inc.) for $20 \mathrm{~min}$ at room temperature in accordance with the manufacturer's instructions. The final siRNA oligonucleotide concentration was $50 \mathrm{nM}$. After $48 \mathrm{~h}$, transfected cells were examined by RT-qPCR as aforementioned or used for further experiments.

Immunohistochemical staining. Immunohistochemical staining of NB tissues was performed using an anti-human TPX2 antibody (1:1,000; cat. no. ab32795; Abcam) as previously described (22).

Western blotting. At $48 \mathrm{~h}$ after transfection, cells were lysed in RIPA buffer (cat. no. F015; BioDynamics Laboratory Inc.) with protease inhibitors, and lysates were centrifuged for $5 \mathrm{~min}$ at $12,000 \times \mathrm{g}$ at $4^{\circ} \mathrm{C}$. The protein concentration was measured using the BCA protein assay kit (cat. no. 23227; Thermo Fisher Scientific, Inc.). Total protein samples $(10 \mu \mathrm{g})$ were separated on $5-20 \%$ gradient polyacrylamide gels (cat. no. c520L; ATTO Corporation) and then transferred 
Table I. Clinicopathological characteristics of patients $(n=43)$.

Clinicopathological charact
Median age of onset, mont
Sex, n (\%)
Male
Female
Primary tumor site, n $(\%)$
Mediastinum
Adrenal gland
Abdomen
Neck

Tumor size, n (\%)

$>5 \mathrm{~cm}$

$\leq 5 \mathrm{~cm}$

Ipsilateral lymph node metastasis, $\mathrm{n}(\%)$

Present

Absent

Contralateral lymph node metastasis, n (\%)

Present

Absent

Distant metastasis, $\mathrm{n}(\%)^{\mathrm{a}}$

Present

Absent

Distant metastasis site, $\mathrm{n}$

Liver

Bone

Marrow

Lymph node

Skin

Complete resection, $\mathrm{n}(\%)$

Yes

No

INSS stage, $\mathrm{n}(\%)$

1

$2 \mathrm{~A}$

2B

3

4

$4 \mathrm{~s}$

MYCN amplification, n (\%)

Present

Absent

Unknown

Pre-operative chemotherapy, n (\%)

Present

Absent

Radiation, n (\%)

Present

Absent

$10(23.3)$

33 (76.7)

PBSCT, n (\%)

Present

Absent

19 (44.2)

12 (27.9)

1 (2.3)

$23(53.5)$

$20(46.5)$

$23(53.5)$

20 (46.5)

$7(16.3)$

36 (83.7)

15 (34.9)

28 (65.1)

7

6

7

6

1

$24(55.8)$

$19(44.2)$

7 (16.3)

7 (16.3)

$6(14.0)$

8 (18.6)

$11(25.6)$

4 (9.3)

7 (16.3)

32 (74.4)

4 (9.3)

12 (27.9)

40 (93.0)
Table I. Continued.

\begin{tabular}{lc}
\hline Clinicopathological characteristic & Value \\
\hline BMT, n (\%) & \\
Present & $4(9.3)$ \\
Absent & $39(90.7)$ \\
Prognosis, n (\%) & \\
Alive & $32(74.4)$ \\
Dead & $11(25.6)$
\end{tabular}

a Due to multiple metastases in certain patients, the number of metastases is greater than the total number of patients with distant metastasis. INSS, International Neuroblastoma Staging System; PBSCT, peripheral blood stem cell transplantation; BMT, bone marrow transplantation; MYCN, MYCN proto-oncogene bHLH transcription factor.

onto a polyvinylidene difluoride filter (cat. no. WSE4050; ATTO Corporation). The filter was first blocked with 5\% skimmed milk for $1 \mathrm{~h}$ at room temperature and then incubated with primary antibodies against TPX2 $(1: 5,000)$ and $\beta$-actin (1:20,000; cat. no. 691001) (both MP Biomedicals, LLC) for $15 \mathrm{~min}$ at room temperature. Following the primary incubation, membranes were incubated with horseradish peroxidase-conjugated anti-mouse IgG antibody (1:10,000 for TPX2 and 1:40,000 for $\beta$-actin) (cat. no. W402B; Promega Corporation) for $30 \mathrm{~min}$ at room temperature. The protein bands were visualized by a WSE-6100 LuminoGraph imaging system (ATTO Corporation).

Immunofluorescence staining. For double immunofluorescence of cell lines and NB tissues, sections were incubated with primary antibodies against TPX2 (1:500) and N-Myc (1:200; cat. no. 51705; Cell signaling Technology, Inc.) overnight at $4^{\circ} \mathrm{C}$. Alexa Fluor ${ }^{\circledR} 546$ goat anti-mouse IgG (1:500; cat. no. A-11030) and Alexa Fluor ${ }^{\circledR} 488$ goat anti-rabbit IgG (1:200; cat. no. A-11008) (both Invitrogen; Thermo Fisher Scientific, Inc.) were used as secondary antibodies for $1 \mathrm{~h}$ at room temperature. Nuclear counterstaining was performed using ProLong ${ }^{\circledR}$ Gold Antifade Reagent with DAPI (cat. no. 727434; Invitrogen; Thermo Fisher Scientific, Inc.). Confocal images were acquired using a BX53 inverted microscope with a DP74 digital camera system (Olympus). Further details are provided in Appendix 1.

Cell proliferation assay. Cell proliferation of TPX2 siRNAand control siRNA-transfected NB cells was evaluated with a Cell Counting Kit-8 (cat. no. CK04; Dojindo Laboratories, Inc.) in accordance with the manufacturer's instructions. Additional experimental details on the assay are provided in Appendix 1.

Cell cycle analysis and apoptosis assay. The DNA content of TPX2 siRNA- and control siRNA-transfected NB cells was evaluated using the Muse ${ }^{\mathrm{TM}}$ Cell Cycle Assay kit (cat. no. MCH100106) and Muse Annexin V \& Dead Cell Kit (cat. no. MCH100105) using the Muse Cell Analyzer (all MilliporeSigma) in accordance with the manufacturer's 
instructions. Additional experimental details on these assays are provided in Appendix 1.

Statistical analysis. Statistical analysis was performed using JMP software version 10 (SAS Institute, Inc.) and MedCalc Statistical Software version 19.1.2 (MedCalc Software bvba). Differences between groups were estimated by one-way ANOVA followed by Tukey's post hoc test, and Wilcoxon rank-sum test was used when appropriate. Two-way repeated measures ANOVA was used for the comparisons of repeated measurements. Pearson's correlation coefficient assay was used to analyze expression correlation. Shapiro-Wilk tests were performed to evaluate the normality of distribution and Levene's tests were conducted to assess the equality of variance for comparable groups. For RT-qPCR data, an unpaired two-tailed Student's t-test was used to calculate significant $\Delta \mathrm{Cq}$ differences between two groups. For time-to-event analyses, survival estimates were calculated using the Kaplan-Meier method and groups were compared using the log-rank test. Receiver operating characteristic (ROC) curves with Youden's index were established to determine the cut-off values for analyzing prognosis. All P-values were calculated using the two-tailed test and $\mathrm{P}<0.05$ was considered to indicate a statistically significant difference.

\section{Results}

Association between patient characteristics and TPX2 expression. Representative immunostaining images of NB tissues from validation set are revealed in Fig. 1A. The median was used as the cut-off point for the definition of high and low TPX2 expression TPX2 mRNA expression was significantly higher in NB with MYCN gene amplification compared with that in patients with a single copy of MYCN in both the TARGET-NB set $(\mathrm{P}<0.0001)$ and the validation set $(\mathrm{P}=0.002)$ (Fig. 1B; Table II). In clinical trials of NB, Aurora kinase inhibitor has been shown to exhibit preclinical activity (24); therefore, the correlation between Aurora kinase A (AURKA) and TPX2 (activator of AURKA) was examined. A significant linear correlation was observed between TPX2 expression and AURKA expression in the TARGET-NB set $\left(r^{2}=0.78\right.$, $\mathrm{P}<0.0001$; Fig. S1A). In the TARGET-NB set, TPX2 expression was significantly associated with age at diagnosis, stage, MYCN status and tumor histology (Table SII).

High TPX2 expression is significantly associated with poor prognosis in patients with $N B$. To evaluate the association between TPX2 expression and the outcome of NB patients, data of NB tumor samples from TARGET (containing complete clinical data and survival information) were analyzed. The cut-off value was defined according to the best predictive value as calculated by ROC analysis (Fig. S1B and C). The cut-off values for TARGET-NB set and the validation set of the present study were set at 8.10 and 13.96 , respectively. It was found that high expression of TPX2 in patients with NB was significantly associated with decreased overall survival (OS) $(\mathrm{P}=0.0008, \log$-rank test; Fig. 1C). Data from the validation set revealed the same result $(\mathrm{P}=0.0037, \log$-rank test) (Fig. 1D). These results showed that high expression of TPX2 in patients with NB was significantly correlated with a poor prognosis for
OS. However, in the multivariate analysis of the TARGET-NB set, only the INSS stage was a strong independent prognostic factor (Table SIII).

Combined assessment of TPX2 expression and MYCN gene amplification. Considering the prognostic role of MYCN gene amplification in NB (25), it was hypothesized that the combined assessment of TPX2 expression (high/low expression) and MYCN status (MYCN amplification +/-) may result in an increased predictive effect. The patients were divided into three groups on the basis of the expression levels of TPX2 and MYCN. It was found that NB patients with high expression of TPX2 and MYCN gene amplification had the poorest prognosis in both the TARGET-NB set and the present validation set (Fig. 1E and F). The colocalization of TPX2 with N-Myc in NB cells was also observed using double fluorescence immunohistochemistry. Similar results were observed in NB tissues (Fig. 2A).

Inhibition of TPX2 suppresses cell proliferation in NB cells. To explore the functional role of TPX2 in NB cells, proliferation was evaluated in the MYCN-amplified cell line (IMR5) and MYCN-non-amplified cell line [KP-N-SI(FA)] with TPX2 knockdown mediated by siRNA. At $48 \mathrm{~h}$ post-transfection of TPX2 siRNA, TPX2 expression was significantly decreased in both the IMR5 and KP-N-SI(FA) cells (Fig. S1D). It was found that NB cell proliferation was significantly decreased following TPX2 knockdown compared with that in controls in both cell lines (Fig. 2B).

Knockdown of TPX2 expression induces $G 2 / M$ arrest in NB cells. Next, the influence of TPX2 on the cell cycle distribution of NB cells was evaluated. After TPX2 knockdown in the MYCN-amplified cell line and MYCN-non-amplified cell line, the percentage of $\mathrm{G} 2 / \mathrm{M}$ cells was increased compared with that in the controls (Fig. 2C).

Inhibition of TPX2 promotes early apoptosis in NB cells. To determine whether increased apoptosis induction may explain the observed phenotype in TPX2 knockdown NB cells, cell death and apoptosis were determined using an Annexin V \& Dead Cell kit. Apoptosis assays revealed that TPX2 knockdown significantly decreased NB cell growth and increased the percentage of early apoptotic cells (Fig. 2D).

\section{Discussion}

In the present study, the prognostic role of TPX2 in NB and the association between TPX2 expression and MYCN gene amplification in NB were evaluated using the TARGET-NB data set and a validation set. It was found that high expression of TPX2 was significantly associated with the poor OS of patients with NB in both data sets. The expression of TPX2 was higher in patients with MYCN gene amplification, and patients with high TPX2 expression and MYCN amplification had the poorest OS compared with patients with either low TPX2 expression or a single copy of MYCN. The colocalization of TPX2 with $\mathrm{N}-\mathrm{Myc}$ in NB cells and tissue was observed. Knockdown of TPX2 in NB cell lines suppressed the proliferation of NB cells and blocked the cell cycle progression. Further analysis 
A

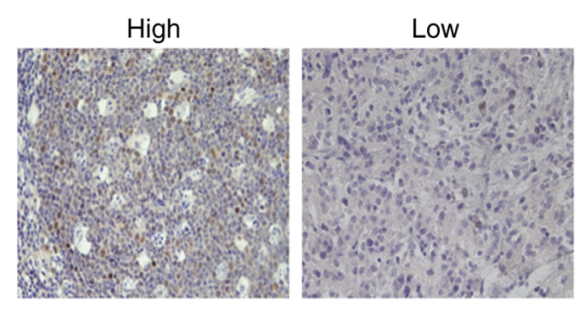

C

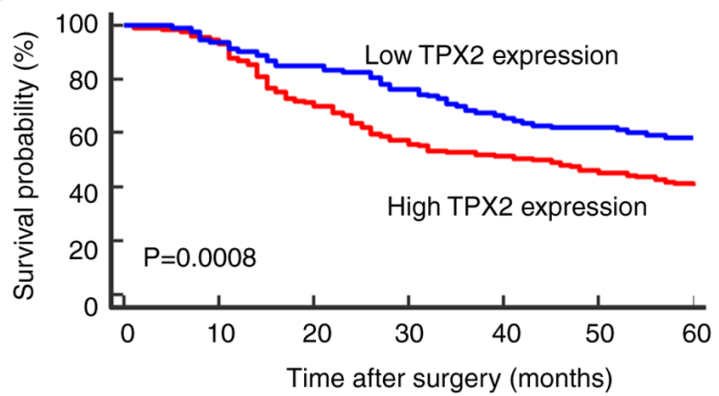

Number at risk

Group: High TPX2 expression

$\begin{array}{rrrrrrr}133 & 123 & 92 & 73 & 67 & 57 & 47 \\ \text { Group: Low TPX2 expression } & & & & \\ 114 & 107 & 97 & 86 & 73 & 68 & 63\end{array}$

E

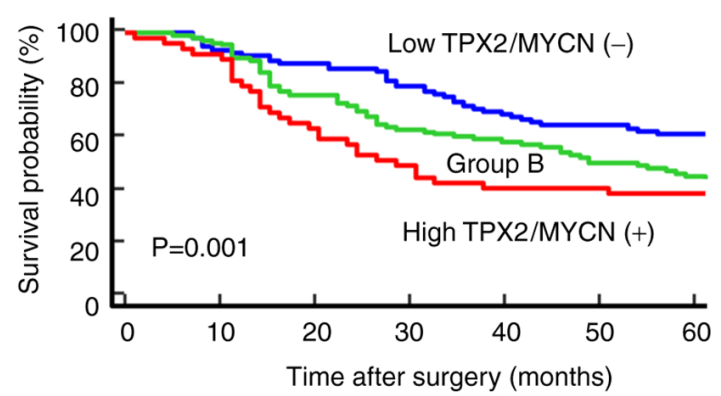

Number at risk

Group A: High TPX2/MYCN (+)

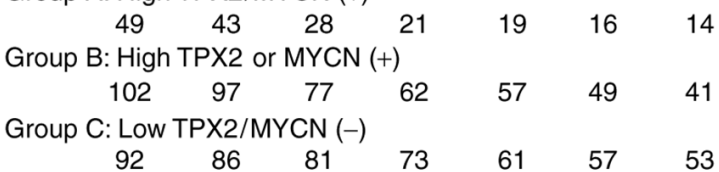
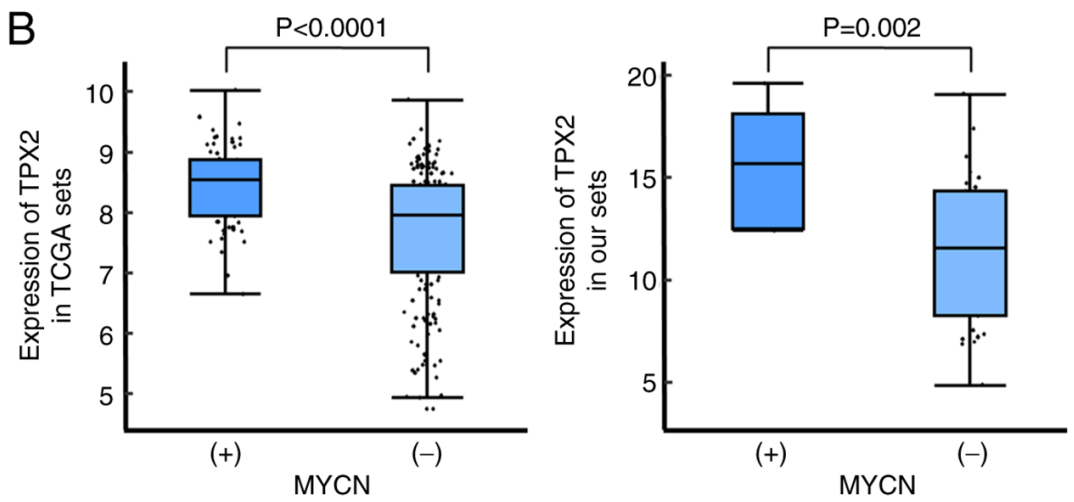

$\mathrm{D}$

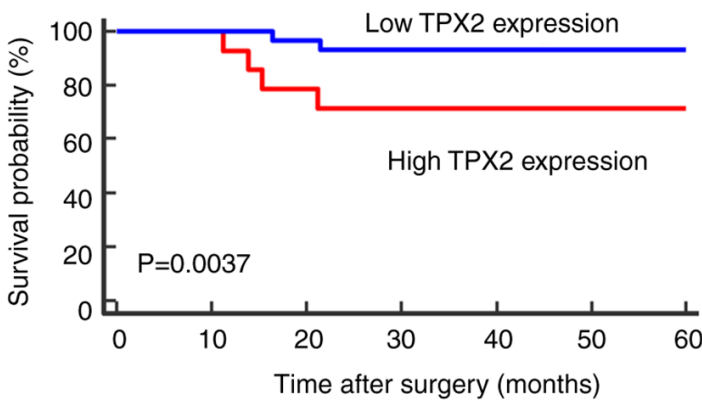

Number at risk

Group: High TPX2 expression

$\begin{array}{rrrrrrr}14 & 14 & 11 & 9 & 9 & 9 & 8 \\ \text { Group: Low TPX2 expression } & & & & \\ 29 & 29 & 28 & 27 & 24 & 24 & 23\end{array}$

$\mathrm{F}$

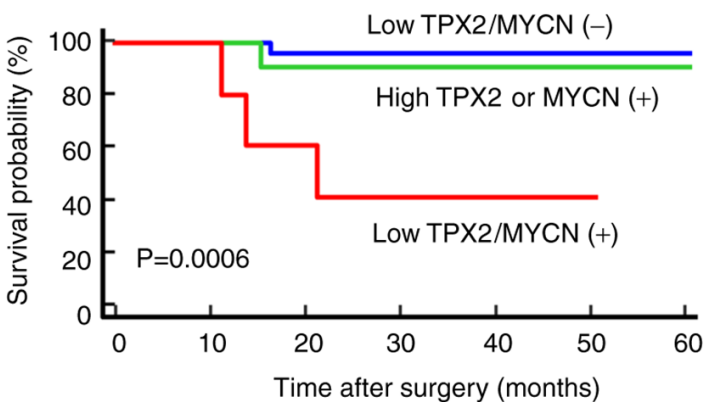

Number at risk

Group A: High TPX2/MYCN (+)

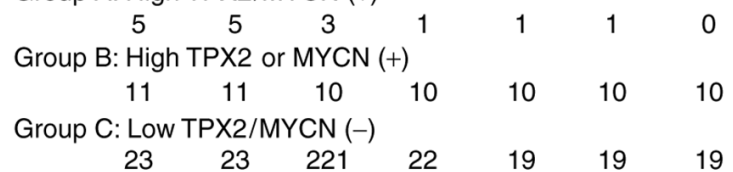

Figure 1. Prognostic effect of TPX2 and the association between TPX2 expression and MYCN gene amplification in NB. (A) Representative immunostaining images of NB tissue with low and high TPX2 expression (x200 magnification). (B) TPX2 expression was significantly higher in NB tumors with MYCN gene amplification compared with that in NB tumors harboring a single copy of MYCN gene in both the TARGET-NB set and the validation set. (C and D) Kaplan-Meier survival curves of patients with NB according to TPX2 expression. The high TPX2 expression group had a significantly poorer prognosis compared with the low TPX2 expression group in both the TARGET-NB set and the validation set. (E and F) NB tumors with high TPX2 expression group combined with MYCN gene amplification displayed the worst prognosis in both the TARGET-NB set and the validation set. Two-tailed t-tests were performed to calculate the P-value. Statistical analysis of the survival was performed using the log-rank test. TPX2, targeting protein for Xenopus kinesin-like protein 2; NB, neuroblastoma; TARGET, Therapeutically Applicable Research to Generate Effective Treatments; MYCN, MYCN proto-oncogene bHLH transcription factor.

indicated that early apoptosis was significantly increased in NB cells after TPX2 knockdown.

TPX2 is a microtubule-associated protein and a critical factor for mitosis and spindle assembly (8). TPX2 recruits and activates AURKA during mitosis $(26,27)$. TPX2 binding and phosphorylation of the Thr288 site of AURKA affect the conformation of AURKA, inducing AURKA activity (28). In addition, TPX 2 enters the nucleus in response to DNA double-strand breaks induced by ionizing radiation and regulates $\gamma$-histone 2AX ( $\gamma$-H2AX) levels in DNA repair (29). 
Table II. Association between patient characteristics and TPX2 expression in the validation set.

\begin{tabular}{|c|c|c|}
\hline $\begin{array}{l}\text { Clinicopathological } \\
\text { characteristic }\end{array}$ & $\begin{array}{l}\text { TPX2 expression } \\
(\text { mean } \pm \text { SD })\end{array}$ & P-value \\
\hline Age of onset, months & & 0.39 \\
\hline$\geq 12$ & $11.3 \pm 3.4$ & \\
\hline$<12$ & $12.3 \pm 4.0$ & \\
\hline Sex & & 0.68 \\
\hline Male & $11.5 \pm 3.6$ & \\
\hline Female & $12.1 \pm 3.9$ & \\
\hline Primary tumor site & & 0.59 \\
\hline Abdomen & $11.2 \pm 3.4$ & \\
\hline Other & $11.9 \pm 3.8$ & \\
\hline Primary tumor size, $\mathrm{cm}$ & & 0.58 \\
\hline$>5$ & $12.0 \pm 4.1$ & \\
\hline$\leq 5$ & $11.4 \pm 3.3$ & \\
\hline Distant metastasis & & 0.45 \\
\hline Present & $12.3 \pm 3.9$ & \\
\hline Absent & $11.4 \pm 3.5$ & \\
\hline Complete resection & & 0.55 \\
\hline Yes & $12.0 \pm 3.7$ & \\
\hline No & $11.3 \pm 3.6$ & \\
\hline INSS stage & & 0.36 \\
\hline $1 / 2 / 3 / 4 s$ & $11.4 \pm 3.3$ & \\
\hline 4 & $12.6 \pm 4.5$ & \\
\hline MYCN amplification & & 0.002 \\
\hline Present & $15.4 \pm 2.6$ & \\
\hline Absent & $11.0 \pm 3.4$ & \\
\hline INPC stage & & 0.96 \\
\hline Favorable & $11.6 \pm 3.3$ & \\
\hline Unfavorable & $11.7 \pm 4.1$ & \\
\hline
\end{tabular}

TPX2, targeting protein for Xenopus kinesin-like protein 2; INSS, International Neuroblastoma Staging System; INPC, International Neuroblastoma Pathology Classification; MYCN, MYCN proto-oncogene bHLH transcription factor.

TPX2 and its partner AURKA are frequently upregulated in human tumors $(8,30)$. Knockdown of TPX2 or AURKA leads to elevated $\gamma-\mathrm{H} 2 \mathrm{AX}(17,31)$, which is associated with increased apoptosis (32). van Gijn et al (33) reported TPX2/AURKA signaling as a potential therapeutic target in genomically unstable cancer cells. However, the most current treatment strategy targeting TPX2/AURKA signaling is only focusing on Aurora kinase inhibitors (34).

Several studies have reported the prognostic role of TPX2 expression in various types of human malignancies, including gastric, colon, hepatic, pancreatic, lung, salivary gland and cervical cancer $(13,15,35-39)$. In colorectal cancer, TPX2 promotes the progression of colorectal adenoma to carcinoma (15). Ognibene et al (17) reported that high TPX2 expression in NB was associated with unfavorable OS and relapse-free survival using Versteeg $(\mathrm{n}=88)$ and SEQC $(\mathrm{n}=498)$ datasets. TPX2 expression in MYCN gene-amplified patients with NB was significantly higher compared with expression in MYCN single copy patients (17). Ooi et al (40) found that spindle assembly genes, including TPX2 and AURKA genes, are upregulation and are predictive of a poor outcome in NB bearing MYCN amplification in high-risk NB patients with MYCN amplification. Another study showed that colon cancer patients with high expression of MYC and AURKA/TPX2 have the poorest OS (41). The present study results were in line with the aforementioned findings. In the present study, colocalization of TPX2 with N-Myc in the nucleus and cytoplasm of NB cells was observed. The findings of the present study also revealed that high TPX2 expression leads to a worse prognosis in NB patients with MYCN amplification. AURKA inhibitor alters the structure of AURKA and results in structural destabilization of the MYC-AURKA complex (42), preventing phospho-MYC-AURKA protein complex formation and leading to N-Myc degradation and cell death in cancer (43). These findings and mechanisms could explain the association of high TPX2 with poor outcome and the increase of TPX2, an activator of AURKA, in MYCN gene-amplified patients with NB.

Knockdown of TPX2 expression using siRNA in multiple cancer cell lines has been shown to significantly inhibit cell proliferation and viability $(39,44,45)$. The results of the present study revealed that proliferation was significantly decreased in both a MYCN-amplified cell line and a MYCN-non-amplified cell line with TPX2 knockdown. Further apoptosis analysis demonstrated that TPX2 may play an anti-apoptotic role in NB cells. Knockdown of TPX2 promotes cell death by decreasing the resistance to apoptosis. The expression of TPX2 is strictly controlled by the cell cycle. During the $S / G_{2}$ phases, TPX2 localizes to mitotic spindle poles and then is degraded after completion of cytokinesis (8). Chu et al (46) found that the Aurora-A/TPX2 molecular axis regulated the cell cycle progression of breast cancer cells. The present results identified that knockdown of TPX2 led to a cell cycle arrest at the $\mathrm{G}_{2} / \mathrm{M}$ phase.

The present study has several limitations. First, it is a single-institution study and the expression of TPX2 was assessed in only 43 NB tissues. Second, due to the long-term span of this retrospective study, it was not possible to collect enough paraffin-embedded tissue sections to assess the expression of TPX2 protein in NB specimens by immunohistochemical staining. Third, the role of TPX2, such as that in DNA repair and chemoresistance, under NB-relevant drugs was not evaluated. Finally, the study did not provide evidence showing interaction between TPX2 and MYCN. Further experimental evidence is necessary to prove the mechanism underlying the effect between TPX2 and MYCN.

In conclusion, the present study provided evidence for the clinical role and biological function of TPX2 in NB. The present findings suggested that clinical assessment of TPX2 expression in primary tumors may provide prognostic information for children with NB, particularly for NB patients with MYCN amplification. Considering the high incidence of NB in childhood tumors and the poor treatment effect, targeting TPX2 may be a novel therapy for NB. 
A
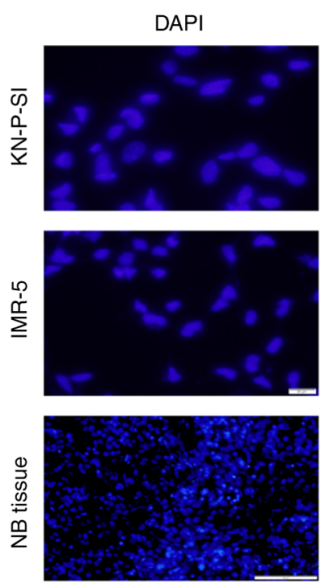
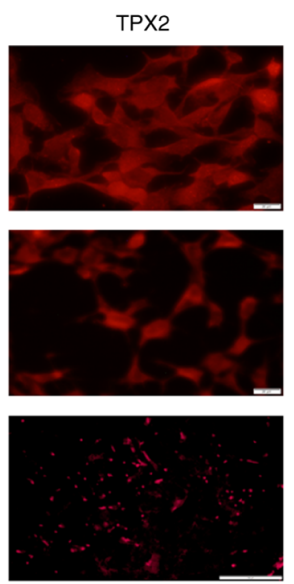
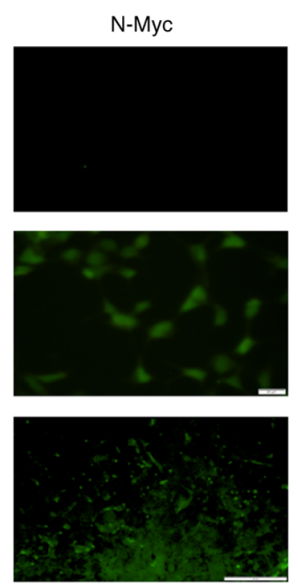

C

IMR5:MYCN (+)
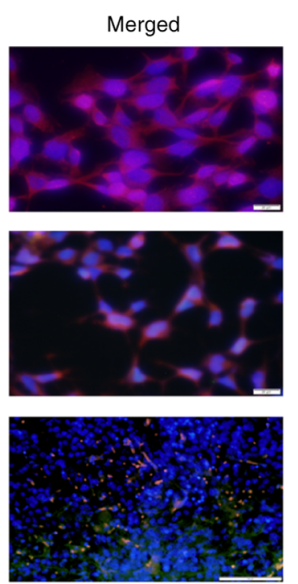

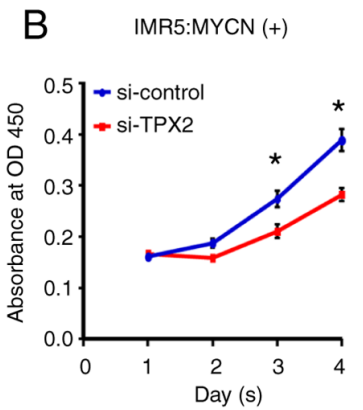

KP-N-SI:MYCN (-)
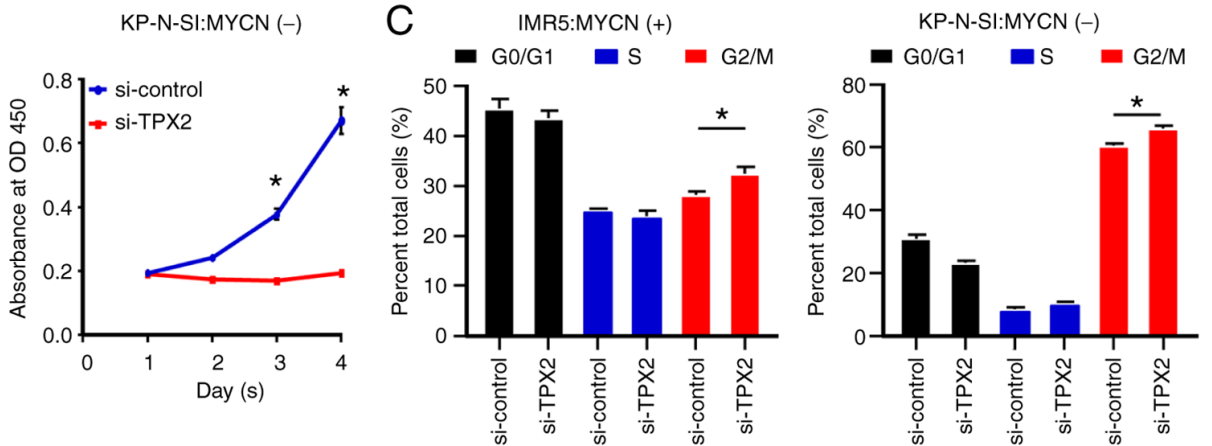

D

IMR-5
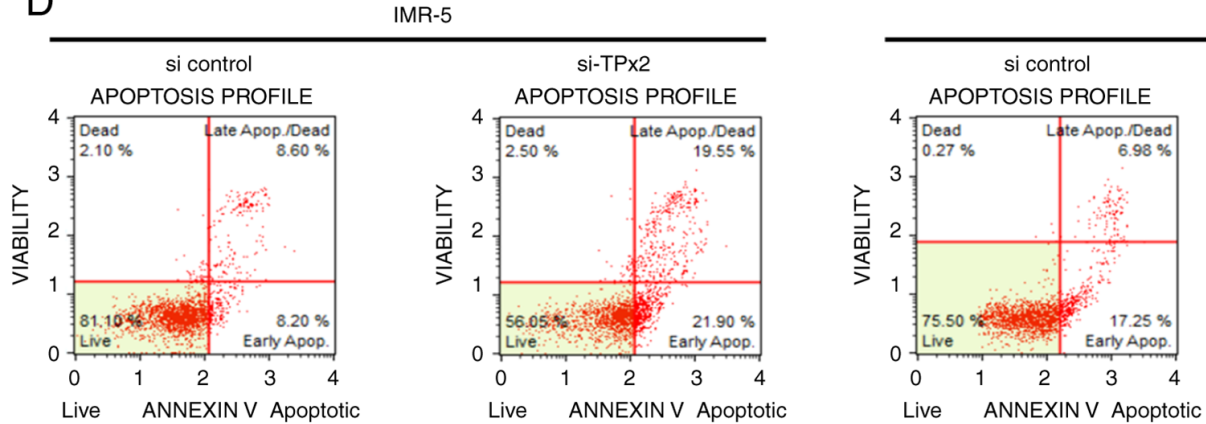

KN-P-SI
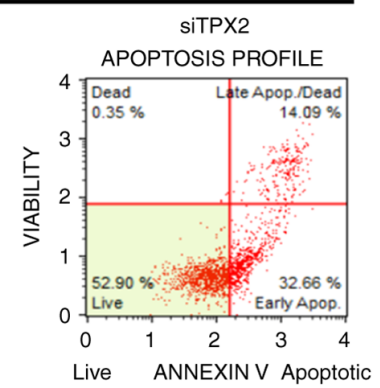

IMR-5
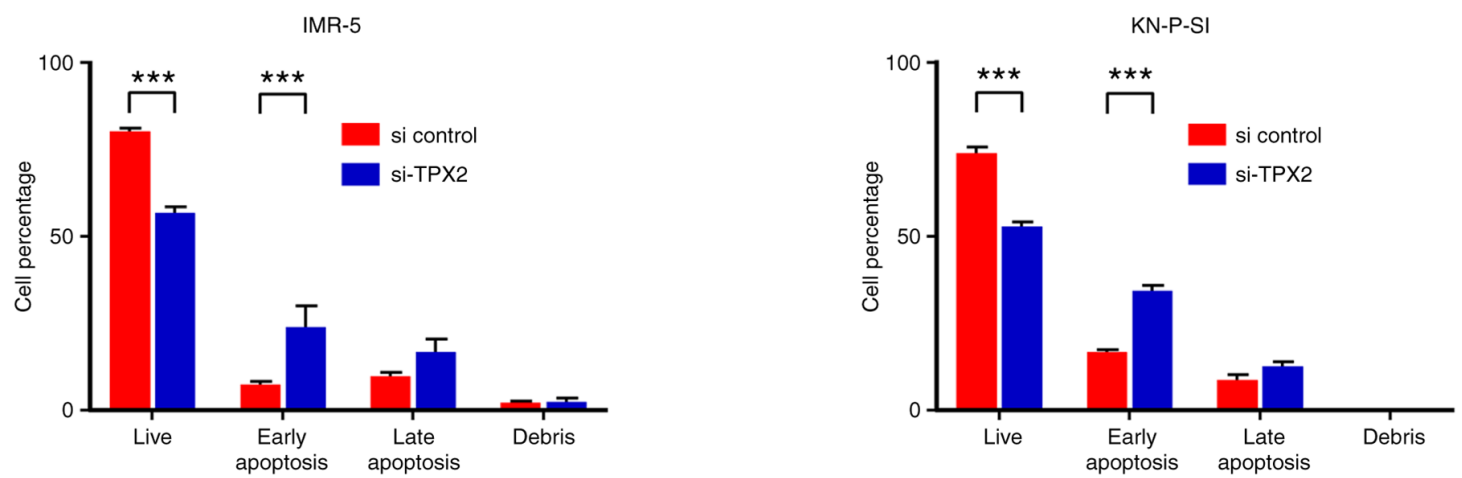

Figure 2. TPX2 downregulation inhibits proliferation, blocks cell cycle progression and decreases the resistance to apoptosis in NB cells. (A) Immunofluorescence staining of NB cells (upper and middle panels; magnification, x400) and tissue (lowest panels; magnification, x400). (B) Cell proliferation was examined in the MYCN-amplified cell line and MYCN-non-amplified cell line transfected with siRNA targeting TPX2 and control siRNA. (C) Analysis of cell cycle distribution was performed in cell lines transfected with siRNA targeting TPX2 and control siRNA. (D) TPX2 knockdown significantly decreased NB cell growth and increased the percentage of early apoptotic cells. Data are presented as the mean \pm SD of three replicates. "P $<0.05$, ${ }^{* * *} \mathrm{P}<0.001$; one-way ANOVA was used to compare groups. TPX2, targeting protein for Xenopus kinesin-like protein 2; NB, neuroblastoma; si/siRNA, small interfering RNA; MYCN, MYCN proto-oncogene bHLH transcription factor.

\section{Acknowledgements}

Not applicable.

\section{Funding}

No funding was received. 


\section{Availability of data and materials}

The datasets used and/or analyzed during the current study are available from the corresponding author on reasonable request.

\section{Authors' contributions}

YK, CY, YT and YOku conceptualized and designed the study. YK, YS, YN, KM, KA, KO, MI, KU and MH provided the samples and clinical information, and helped analyze results. YK, CY, YT, YOki, YOku, AY, TK, TS, MK and MO analyzed and interpreted the data. YK, CY, YT and YOku performed statistical analysis. CY, YK, YT and YOku drafted the manuscript. All authors revised the manuscript for important intellectual content. YK and CY confirm the authenticity of all the raw data. All authors read and approved the final manuscript.

\section{Ethics approval and consent to participate}

The protocol for this research project was approved (approval no. 1464) by the Institutional Review Board of Mie University Hospital (Tsu, Japan). The study was conducted in accordance with The Declaration of Helsinki. Informed consent was obtained from the parents/guardians of the patients using the opt-out scheme.

\section{Patient consent for publication}

Not applicable.

\section{Competing interests}

The authors declare that they have no competing interests.

\section{References}

1. Pizzo PA PD: Principles and Practice of Pediatric Oncology Sixth edition. Wolters Kluwer Health/Lippincott Williams and Wilkins. Philadelphia, 2010.

2. Smith MA, Altekruse SF, Adamson PC, Reaman GH and Seibel NL: Declining childhood and adolescent cancer mortality. Cancer 120: 2497-2506, 2014.

3. American Cancer Society. Key Statistics About Neuroblastoma. https://www.cancer.org/cancer/neuroblastoma/about/key-statistics.html. Accessed December 17, 2021.

4. Siegel RL, Miller KD, Fuchs HE and Jemal A: Cancer Statistics, 2021. CA Cancer J Clin 71: 7-33, 2021.

5. Park JR, Kreissman SG, London WB, Naranjo A, Cohn SL, Hogarty MD, Tenney SC, Haas-Kogan D, Shaw PJ, Kraveka JM, et al: Effect of tandem autologous stem cell transplant vs single transplant on event-free survival in patients with high-risk neuroblastoma: A randomized clinical trial. JAMA 322: 746-755, 2019.

6. Matthay KK, Maris JM, Schleiermacher G, Nakagawara A, Mackall CL, Diller L and Weiss WA: Neuroblastoma. Nat Rev Dis Primers 2: 16078, 2016.

7. Wieczorek M, Bechstedt S, Chaaban S and Brouhard GJ: Microtubule-associated proteins control the kinetics of microtubule nucleation. Nat Cell Biol 17: 907-916, 2015.

8. Neumayer G, Belzil C, Gruss OJ and Nguyen MD: TPX2: Of spindle assembly, DNA damage response, and cancer. Cell Mol Life Sci 71: 3027-3047, 2014.

9. Heidebrecht HJ, Buck F, Steinmann J, Sprenger R, Wacker HH and Parwaresch R: p100: A novel proliferation-associated nuclear protein specifically restricted to cell cycle phases S, G2, and M. Blood 90: 226-233, 1997.
10. Garrido G and Vernos I: Non-centrosomal TPX2-dependent regulation of the Aurora a kinase: Functional implications for healthy and pathological cell division. Front Oncol 6: 88, 2016.

11. Moss DK, Wilde A and Lane JD: Dynamic release of nuclear RanGTP triggers TPX2-dependent microtubule assembly during the apoptotic execution phase. J Cell Sci 122(Pt 5): 644-655, 2009.

12. Yan L, Li Q, Yang J and Qiao B: TPX2-p53-GLIPR1 regulatory circuitry in cell proliferation, invasion, and tumor growth of bladder cancer. J Cell Biochem 119: 1791-1803, 2018.

13. Tomii C, Inokuchi M, Takagi Y, Ishikawa T, Otsuki S, Uetake H, Kojima $\mathrm{K}$ and Kawano T: TPX2 expression is associated with poor survival in gastric cancer. World J Surg Oncol 15: 14, 2017.

14. Zhu H, Liu J, Feng J, Zhang Q, Bian T, Li X, Sun H, Zhang J and Liu Y: Overexpression of TPX2 predicts poor clinical outcome and is associated with immune infiltration in hepatic cell cancer. Medicine (Baltimore) 99: e23554, 2020.

15. Sillars-Hardebol AH, Carvalho B, Tijssen M, Beliën JA, de Wit M, Delis-van Diemen PM, Pontén F, van de Wiel MA, Fijneman RJ and Meijer GA: TPX2 and AURKA promote 20q amplicon-driven colorectal adenoma to carcinoma progression. Gut 61: 1568-1575, 2012.

16. Shah KN, Bhatt R, Rotow J, Rohrberg J, Olivas V, Wang VE, Hemmati G, Martins MM, Maynard A, Kuhn J, et al: Aurora kinase A drives the evolution of resistance to third-generation EGFR inhibitors in lung cancer. Nat Med 25: 111-118, 2019.

17. Ognibene M, Podestà M, Garaventa A and Pezzolo A: Role of GOLPH3 and TPX2 in neuroblastoma DNA damage response and cell resistance to chemotherapy. Int J Mol Sci 20: 4764, 2019.

18. Irwin MS, Naranjo A, Zhang FF, Cohn SL, London WB, Gastier-Foster JM, Ramirez NC, Pfau R, Reshmi S, Wagner E, et al: Revised neuroblastoma risk classification system: A report from the children's oncology group. J Clin Oncol 39: 3229-3241, 2021.

19. Bosse KR and Maris JM: Advances in the translational genomics of neuroblastoma: From improving risk stratification and revealing novel biology to identifying actionable genomic alterations. Cancer 122: 20-33, 2016.

20. Cohn SL, Pearson AD, London WB, Monclair T, Ambros PF, Brodeur GM, Faldum A, Hero B, Iehara T, Machin D, et al: The International neuroblastoma risk group (INRG) classification system: An INRG Task Force report. J Clin Oncol 27: 289-297, 2009.

21. Nakagawara A, Li Y, Izumi H, Muramori K, Inada $\mathrm{H}$ and Nishi M: Neuroblastoma. Jpn J Clin Oncol 48: 214-241, 2018.

22. Matsushita K, Uchida K, Saigusa S, Ide S, Hashimoto K, Koike Y, Otake K, Inoue M, Tanaka K and Kusunoki M: Glycolysis inhibitors as a potential therapeutic option to treat aggressive neuroblastoma expressing GLUT1. J Pediatr Surg 47: 1323-1330, 2012.

23. Livak KJ and Schmittgen TD: Analysis of relative gene expression data using real-time quantitative PCR and the 2(-Delta Delta C(T)) method. Methods 25: 402-408, 2001.

24. Mossé YP, Fox E, Teachey DT, Reid JM, Safgren SL, Carol H, Lock RB, Houghton PJ, Smith MA, Hall D, et al: A phase II study of alisertib in children with recurrent/refractory solid tumors or leukemia: Children's oncology group phase I and pilot consortium (ADVL0921). Clin Cancer Res 25: 3229-3238, 2019.

25. Tonini GP, Boni L, Pession A, Rogers D, Iolascon A, Basso G, Cordero di Montezemolo L, Casale F, Pession A, Perri P, et al: MYCN oncogene amplification in neuroblastoma is associated with worse prognosis, except in stage 4s: The Italian experience with 295 children. J Clin Oncol 15: 85-93, 1997.

26. Bayliss R, Sardon T, Vernos I and Conti E: Structural basis of Aurora-A activation by TPX2 at the mitotic spindle. Mol Cell 12: 851-862, 2003.

27. Asteriti IA, Rensen WM, Lindon C, Lavia P and Guarguaglini G: The Aurora-A/TPX2 complex: A novel oncogenic holoenzyme? Biochim Biophys Acta 1806: 230-239, 2010.

28. Lake EW, Muretta JM, Thompson AR, Rasmussen DM, Majumdar A, Faber EB, Ruff EF, Thomas DD and Levinson NM: Quantitative conformational profiling of kinase inhibitors reveals origins of selectivity for Aurora kinase activation states. Proc Natl Acad Sci USA 115: e11894-e11903, 2018.

29. Neumayer G, Helfricht A, Shim SY, Le HT, Lundin C, Belzil C, Chansard M, Yu Y, Lees-Miller SP, Gruss OJ, et al: Targeting protein for xenopus kinesin-like protein 2 (TPX2) regulates $\gamma$-histone 2AX $(\gamma$-H2AX) levels upon ionizing radiation. J Biol Chem 287: 42206-42222, 2012. 
30. Pérez de Castro I and Malumbres M: Mitotic stress and chromosomal instability in cancer: The case for TPX2. Genes Cancer 3: 721-730, 2012.

31. Do TV, Hirst J, Hyter S, Roby KF and Godwin AK: Aurora A kinase regulates non-homologous end-joining and poly(ADP-ribose) polymerase function in ovarian carcinoma cells. Oncotarget 8: 50376-50392, 2017.

32. Liu Y, Parry JA, Chin A, Duensing S and Duensing A: Soluble histone $\mathrm{H} 2 \mathrm{AX}$ is induced by DNA replication stress and sensitizes cells to undergo apoptosis. Mol Cancer 7: 61, 2008.

33. van Gijn SE, Wierenga E, van den Tempel N, Kok YP, Heijink AM, Spierings DCJ, Foijer F, van Vugt MATM and Fehrmann RSN: TPX2/Aurora kinase A signaling as a potential therapeutic target in genomically unstable cancer cells. Oncogene 38 852-867, 2019.

34. Niu H,Manfredi M and Ecsedy JA: Scientific rationale supporting the clinical development strategy for the investigational Aurora a kinase inhibitor alisertib in cancer. Front Oncol 5: 189, 2015.

35. Ma Y, Lin D, Sun W, Xiao T, Yuan J, Han N, Guo S, Feng X, Su K, Mao Y, et al: Expression of targeting protein for xklp2 associated with both malignant transformation of respiratory epithelium and progression of squamous cell lung cancer. Clin Cancer Res 12: 1121-1127, 2006.

36. Liang B, Jia C, Huang Y, He H, Li J, Liao H, Liu X, Liu X, Bai X and Yang D: TPX2 level correlates with hepatocellular carcinoma cell proliferation, apoptosis, and EMT. Dig Dis Sci 60: 2360-2372, 2015.

37. Warner SL, Stephens BJ, Nwokenkwo S, Hostetter G, Sugeng A, Hidalgo M, Trent JM, Han H and Von Hoff DD: Validation of TPX2 as a potential therapeutic target in pancreatic cancer cells. Clin Cancer Res 15: 6519-6528, 2009.

38. Shigeishi H, Ohta K, Hiraoka M, Fujimoto S, Minami M, Higashikawa $\mathrm{K}$ and Kamata N: Expression of TPX2 in salivary gland carcinomas. Oncol Rep 21: 341-344, 2009.

39. Chang H, Wang J, Tian Y, Xu J, Gou X and Cheng J: The TPX2 gene is a promising diagnostic and therapeutic target for cervical cancer. Oncol Rep 27: 1353-1359, 2012.
40. Ooi WF, Re A, Sidarovich V, Canella V, Arseni N, Adami V, Guarguaglini G, Giubettini M, Scaruffi P, Stigliani S, et al: Segmental chromosome aberrations converge on overexpression of mitotic spindle regulatory genes in high-risk neuroblastoma. Genes Chromosomes Cancer 51: 545-556, 2012.

41. Takahashi Y, Sheridan P, Niida A, Sawada G, Uchi R, Mizuno H, Kurashige J, Sugimachi K, Sasaki S, Shimada Y, et al: The AURKA/TPX2 axis drives colon tumorigenesis cooperatively with MYC. Ann Oncol 26: 935-942, 2015.

42. Richards MW, Burgess SG, Poon E, Carstensen A, Eilers M, Chesler L and Bayliss R: Structural basis of N-Myc binding by Aurora-A and its destabilization by kinase inhibitors. Proc Natl Acad Sci USA 113: 13726-13731, 2016.

43. Dauch D, Rudalska R, Cossa G, Nault JC, Kang TW, Wuestefeld T, Hohmeyer A, Imbeaud S, Yevsa T, Hoenicke L, et al: A MYC-Aurora kinase A protein complex represents an actionable drug target in p53-altered liver cancer. Nat Med 22: 744-753, 2016.

44. Wei P, Zhang N, Xu Y, Li X, Shi D, Wang Y, Li D and Cai S: TPX2 is a novel prognostic marker for the growth and metastasis of colon cancer. J Transl Med 11: 313, 2013.

45. Huang Y, Guo W and Kan H: TPX2 is a prognostic marker and contributes to growth and metastasis of human hepatocellular carcinoma. Int J Mol Sci 15: 18148-18161, 2014.

46. Chu TLH, Connell M, Zhou L, HeZ, Won J, Chen H, Rahavi SMR, Mohan P, Nemirovsky O, Fotovati A, et al: Cell cycle-dependent tumor engraftment and migration are enabled by Aurora-A. Mol Cancer Res 16: 16-31, 2018

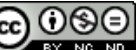

This work is licensed under a Creative Commons Attribution-NonCommercial-NoDerivatives 4.0 International (CC BY-NC-ND 4.0) License. 